\title{
A methodical approach and computer programs to determine the power of active harmonic filters
}

\author{
Bui Ngoc Hung ${ }^{2}$, Lidiia I. Kovernikova ${ }^{1,2^{*}}$ \\ ${ }^{1}$ Melentiev Energy Systems Institute SB RAS, Irkutsk, Russia \\ ${ }^{2}$ Irkutsk National Research Technical University, Irkutsk, Vietnam
}

\begin{abstract}
Poor power quality creates big problems. With the poor power quality, the service life of the equipment is reduced. In connection with the creation and development of intelligent electrical power systems and the digital economy, the requirements for the power quality are increasing. Active harmonic filters began to used to solve problem with the poor power quality. The paper presents a methodical approach and computer programs for determining the power of the active harmonic filter. The power supply system of the Vietnamese coal sorting plant is considered to develop the methodical approach. The results of measurements of the power quality indices and the results of calculating the parameters of the modes of the electrical network of the plant at the fundamental frequency were used. To process a large amount of measured information, the special computer program was developed. On the basis of the methodical approach, the results of measurements of the power quality indices and the computer complex "Stationary permissible optimal mode", the algorithm and the computer program have been developed to calculate the power of the active harmonic filter. The power of the active harmonic filter for the coal sorting plant was determined with the help of this program.
\end{abstract}

\section{Introduction}

Poor power quality creates huge problems for both power supply companies and consumers. Currently, there are deviations from the regulatory requirements for the voltage value, voltage waveform, and balance of three-phase voltages [1-3]. When the power quality is poor, the service life of equipment shortens and additional economic damage occurs [4-6]. Due to the creation and development of intelligent electrical power systems and the digital economy, requirements for power quality are increasing. Active harmonic filters have become adopted to solve problems with the value and non-sinusoidal form of the voltage [7-8]. The paper presents the methodical approach to the selection of active harmonic filters, the computer program to analyze the results of measurements of the power quality indices and operation mode parameters, and the computer program to calculate the power of the active harmonic filter. The methodical approach and programs are illustrated by their use for the selection of the active harmonic filter for the power supply system of the coal grading plant of the Vietnamese company "Cua OngVinacomin".

\section{Methodical approach to the selection of the active harmonic filter}

This includes the six steps presented below.

1) Analysis of power quality indices and operation mode parameters at the point of transmission

\footnotetext{
* Corresponding author: kovernikova@isem.irk.ru
}

of electrical energy or the node of connection of the power supply system of the enterprise to the electrical supply network, where the installation of the active harmonic filter is assumed.

Measurements of power quality indices and operation mode parameters should be carried out in compliance with current regulatory documents [1-2]. Since active harmonic filters are installed to compensate for reactive power and eliminate current harmonics, it is necessary to analyze the following coefficients: voltage value deviation $( \pm \delta U)$, total harmonic distortion of voltage and current $\left(K_{U}, K_{I}\right)$, coefficients of the $n$-th harmonic component of voltage and current $\left(K_{U(n)}, K_{I(n)}\right)$, coefficients of voltage and current interharmonic components $\left(K_{U(m+1 / 2)}, \quad K_{2 U I(m+1 / 2)}\right), \quad$ the negative sequence voltage unbalance coefficient $\left(K_{2 U}\right)$. Other parameters may be considered as well. For example, if necessary, the analysis of the load power factor $(\cos \varphi)$ can be performed.

At the coal sorting plant, measurements of power quality indices and operation mode parameters were carried out at the node where the electrical network of the enterprise was connected to the network of the power supply organization on the $0.4 \mathrm{kV}$ side of the supply transformer (Fig. 1). 


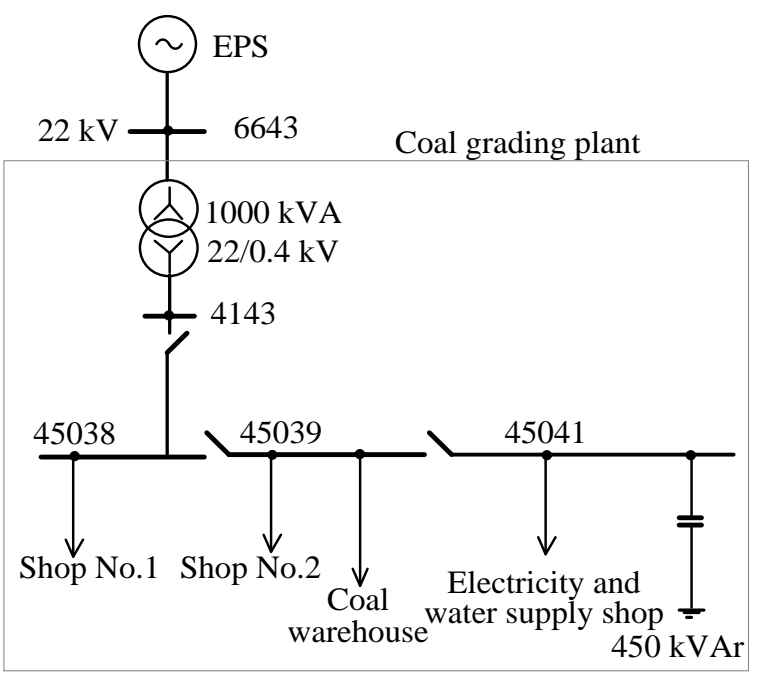

Fig. 1. Power supply system of the coal grading plant.

Tables 1 presents the results of the analysis of measurements of $\pm \delta U, K_{U}, K_{2 U}, \cos \varphi$, and their standard values [2]. Values that fail to comply with the regulatory requirements are shown in bold. Tables 2 presents the results of the analysis of measurements of $K_{U(n)}, K_{I(n)}$. In accordance with [2], the measured values of $K_{U(n)}$ should be less than or equal to $3 \%$, and $K_{I(n)} 12 \%$.

Table 1. Measured values of $\delta U, K_{U}, \cos \varphi, K_{2 U}$, and their standard values.

\begin{tabular}{|l|c|c|c|}
\hline Parameter & Phase A & Phase B & Phase C \\
\hline$\delta U_{\max }, \%$ & 4.1 & 4.5 & 4.6 \\
\hline$\delta U_{\min }, \%$ & -1.3 & -1.7 & -1.4 \\
\hline$\delta U_{\text {std }}, \%$ & \multicolumn{3}{|c|}{$\leq \pm 5,0$} \\
\hline$K_{\text {Umax }}, \%$ & $\mathbf{1 6 . 8}$ & $\mathbf{1 5 . 3}$ & $\mathbf{1 7 . 1}$ \\
\hline$K_{U s t d}, \%$ & $\leq 6.5$ \\
\hline $\cos \varphi_{\max }$, p.u. & 0.98 & 0.92 & 0.99 \\
\hline $\cos \varphi_{\min }$, p.u. & 0.89 & $\mathbf{0 . 7 2}$ & 0.89 \\
\hline $\cos \varphi_{\text {std }}$, p.u. & \multicolumn{3}{|c|}{$\geq 0.85$} \\
\hline$K_{2 \text { max }}, \%$ & \multicolumn{3}{|c|}{0.4} \\
\hline$K_{2 \text { std }}, \%$ & 5.0 \\
\hline
\end{tabular}

Table 2. Measured values of $K_{U(n)}, K_{I(n)}, \%$.

\begin{tabular}{|c|c|c|c|c|c|c|}
\hline$n$ & 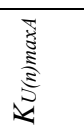 & 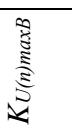 & 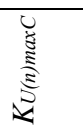 & 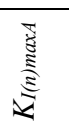 & 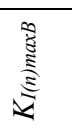 & 咅 \\
\hline 3 & 2.5 & 3.9 & 2.0 & 7.1 & 3.9 & 3.6 \\
\hline 5 & 12.7 & 12.3 & 12.3 & 6.8 & 4.2 & 4.9 \\
\hline 7 & 12.8 & 11.8 & 12.8 & 6.7 & 1.7 & 3.3 \\
\hline 9 & 1.9 & 2.8 & 3.3 & 2.9 & 1.5 & 1.6 \\
\hline 11 & 4.6 & 4.6 & 3.6 & 3.9 & 0.8 & 1.2 \\
\hline 13 & 2.1 & 3.1 & 2.8 & 1.9 & 1.1 & 1.7 \\
\hline 17 & 3.3 & 2.7 & 3.4 & 2.3 & 0.5 & 1.5 \\
\hline 19 & 1.6 & 3.0 & 2.6 & 1.9 & 0.4 & 1.0 \\
\hline 21 & 2.1 & 2.3 & 1.5 & 2.3 & 0.2 & 0.6 \\
\hline 23 & 3.1 & 2.8 & 3.4 & 2.7 & 0.4 & 1.2 \\
\hline 25 & 1.9 & 2.5 & 2.6 & 2.4 & 0.4 & 0.9 \\
\hline
\end{tabular}

Tables 3 presents the results of the analysis of measurements of $K_{U(m+1 / 2)}$ and $I_{(m+1 / 2)}$. The standard values for them have not been established.

Table 3. Measured values of $K_{U(m+1 / 2)}$, and $I_{(m+1 / 2)}$.

\begin{tabular}{|c|c|c|c|c|c|c|}
\hline$\underset{⿱ 亠}{\stackrel{ \pm}{ \pm}}$ & 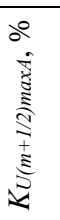 & 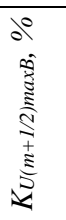 & 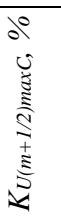 & 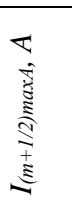 & 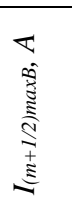 & 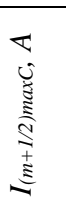 \\
\hline 1.5 & 3.9 & 3.3 & 3.8 & 16.6 & 17.2 & 18.0 \\
\hline 3.5 & 1.1 & 0.8 & 0.9 & 6.0 & 4.8 & 7.3 \\
\hline 5.5 & 1.7 & 1.9 & 0.9 & 5.1 & 5.0 & 6.4 \\
\hline 7.5 & 1.8 & 1.9 & 1.2 & 4.3 & 2.7 & 5.3 \\
\hline 11.5 & 1.3 & 1.7 & 1.4 & 5.1 & 3.7 & 5.0 \\
\hline 13.5 & 1.3 & 1.0 & 0.9 & 5.1 & 2.2 & 5.2 \\
\hline 17.5 & 1.2 & 1.0 & 1.7 & 7.5 & 1.9 & 9.2 \\
\hline 19.5 & 1.5 & 1.3 & 1.7 & 8.5 & 2.7 & 9.7 \\
\hline 21.5 & 2.3 & 2.3 & 1.7 & 9.6 & 3.2 & 7.5 \\
\hline 23.5 & 2.9 & 2.5 & 1.3 & 13.1 & 3.0 & 5.7 \\
\hline 25.5 & 2.2 & 1.5 & 1.5 & 12.5 & 2.7 & 6.5 \\
\hline
\end{tabular}

2) Selection of the type of the active harmonic filter. There are two types of active harmonic filters that differ in purpose and the way they are connected to the network: serial and parallel filters [9-10]. The serial active harmonic filter is used to compensate for current harmonics and to make balancing adjustments of threephase voltages. The parallel filter is used to compensate for reactive power and eliminate current harmonics. It follows from the analysis of the measurement results shown in Tables 1-3 that $\delta U, K_{U}, K_{U(n)}$ exceed the standard values, and $K_{2 U}$ and $\cos \varphi$ are less than the established standard values. It follows from the results of the analysis that to solve the existing problems in the electrical network of the coal grading plant, it is necessary to install the parallel active harmonic filter.

3) Analysis of the regulation capability of transformers supplying the electrical power supply of the enterprise to maintain the required values of voltages.

The goal is to make an analysis of the supply voltage regulation range of the power supply system of the enterprise with the help of the regulating taps of the transformer to achieve the desired voltage value according to the principle of counterload voltage control.

For example, the transformer, through which electrical energy is supplied to the power supply system of the coal grading plant, has 5 regulating taps: $0.38 \mathrm{kV}$; $0.39 \mathrm{kV} ; 0.4 \mathrm{kV} ; 0.41 \mathrm{kV} ; 0.42 \mathrm{kV}$ that allow changing the transformer ratio. The possibilities of voltage regulation by changing the transformer ratio must be taken into account when determining the power of the active harmonic filter.

4) Selection of the node to install the active harmonic filter.

The node to install the active harmonic filter can be designated based on the capabilities and characteristics of the network diagram of the power supply system of 
the enterprise and switchgear. If there are no constraints, then all nodes of the electrical network of the enterprise can be considered as candidates for installation of the active harmonic filter. In either case, an array of candidate nodes is formed. A specific node in this array is determined by solving the optimization problem or in some other way.

At the coal grading plant, node 45038 (Fig. 1) is designated for the installation of the active harmonic filter based on the location and configuration of the 0.4 $\mathrm{kV}$ switchgear that serves electricity to all loads of the enterprise. Node 45038 is located 60 meters away from node 4143 , where measurements of the power quality are made, and the electrical meter is installed.

5) Determining the power of the active harmonic filter.

For the power supply system of the coal grading plant, the power of the active harmonic filter is determined as a result of solving the optimization problem. Its objective function is a minimum of active power losses in the electrical network of the power supply system under the constraints set on $\delta U, K_{U}, K_{U(n)}$, $K_{I(n)}$, and $\cos \varphi$ [2]. Objective function

$$
\sum_{n=1}^{N} \Delta P_{(n)}+\sum_{m=1}^{M-1} \Delta P_{(m+1 / 2)}=\min
$$

where $N$ - the largest harmonic number, $M-1-$ the largest interharmonic number, $\Delta P$ - active power losses in the electrical network subject to the following constraints

$$
\begin{gathered}
0.95 \leq \cos \varphi \leq 1.0, \\
U_{\min } \leq U_{i} \leq U_{\max }, \\
K_{U(n) \min } \leq K_{U(n) A F} \leq K_{U(n) \max }, \\
K_{U \min } \leq K_{A F} \leq K_{U \max }, \\
K_{I(n) \min } \leq K_{I(n) A F} \leq K_{I(n) \max },
\end{gathered}
$$

where $i$ is the network node number, $A F$ - the active harmonic filter. Constraint (2) on $\cos \varphi$ is set by the coal company that owns the coal grading plant.

6) Evaluation of the performance of the selected filter for different modes of operation of the enterprise.

One can evaluate the performance of the selected filter by calculating the parameters of the network at the fundamental frequency (the first harmonic) and at the frequencies of the harmonic components for $n>1$. The power of the active harmonic filter for the coal grading plant was selected as the result of multivariate calculations of the parameters of the mode on of the electrical network at the fundamental frequency. As the result, we have selected the variant of the active harmonic filter power corresponding to the minimum active power loss in the network. To assess the performance of the power of the active harmonic filter at harmonic frequencies given $n>1$, it is also possible to perform operation mode calculations if the electrical networks are long and their lengths are comparable with the voltage and current wavelengths at the frequencies of these harmonics. Since the length of all electrical networks of the enterprise is about $12 \mathrm{~km}$, the wave effect will not manifest itself even at the 50-th harmonic. Therefore, to determine the power of the active harmonic filter to eliminate harmonics, it is sufficient to use the results of measurements of harmonic voltages and currents at the node of the enterprise connection to the supply network.

\section{Program for analyzing the results of measurements of power quality indices and operation mode parameters}

Measurements of power quality indices were performed for 24 hours at 1 -second intervals using a PQ-Box 150 device [11]. The array of each measured parameter consists of 86,400 elements, which poses a significant difficulty for processing and analysis, requiring a large amount of time. The computer program was developed to facilitate the process of analyzing the measurement results [12]. The program is implemented in the MS Excel and Visual Basic programming environment for Windows applications. The program consists of six sheets. Fig. 2 shows the screenshot of Sheet 1 of the program. On the "Main" sheet (Sheet 1) of the program, one enters the measured values in the form of arrays of power quality indices and operation mode parameters at the measuring point. The information can also be deleted and replaced by other information. From the first sheet of the program, one can navigate to the other five sheets.

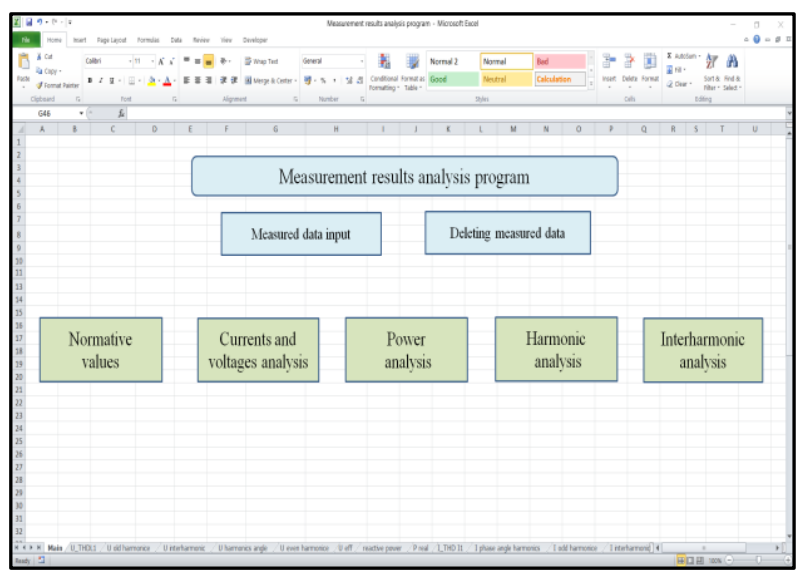

Fig. 2. Sheet 1 of the program for analyzing measurement results.

Sheet 2 provides information on the standard values set for the values of power quality indices and $\cos \varphi$ in the regulatory documents in Vietnam. On Sheet 3, the analysis of currents and voltages is performed: it outputs plots of $\delta U$ changes for three phases and standard values of $\pm \delta U$, the table with maximum and minimum values of $\delta U$ and the standard value; the plots of the effective values of the phase currents and statistical estimates of the values of the currents are displayed. Fig. 3 shows a screenshot of Sheet 3, which shows plots of changes in $\delta U$ values in three phases over the measurement time and the standard values.

Sheet 4 analyzes the active and reactive powers and load power factor: it displays the plots of active power, reactive power, and $\cos \varphi$ in three phases during the 
measurement time and tables of statistical estimates of these parameters.

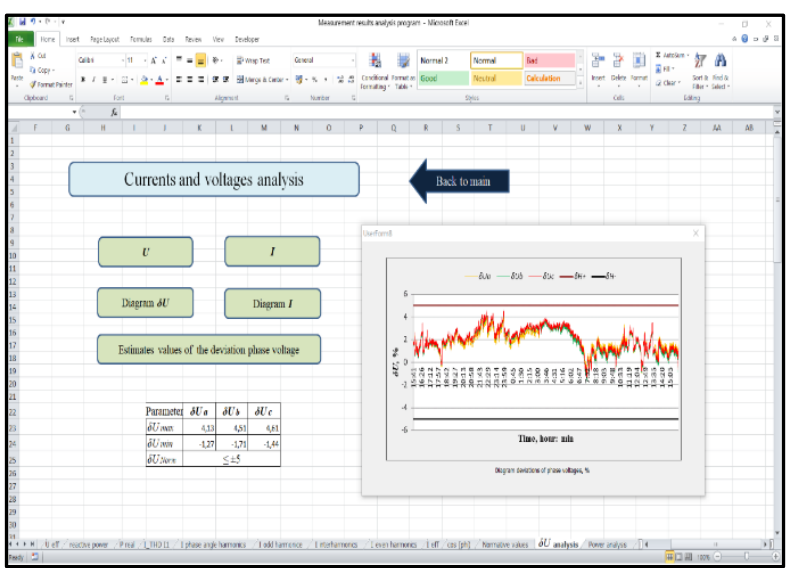

Fig. 3. Sheet 3 of the program for the analysis of measurement results.

Sheet 5 (Fig. 4) analyzes the current and voltage harmonics: the program outputs the plots of changes in the total harmonic distortion of current and voltage and the table of their statistical estimates; the tables of statistical estimates are displayed for coefficients of the $n$-th harmonic component of voltage and current.

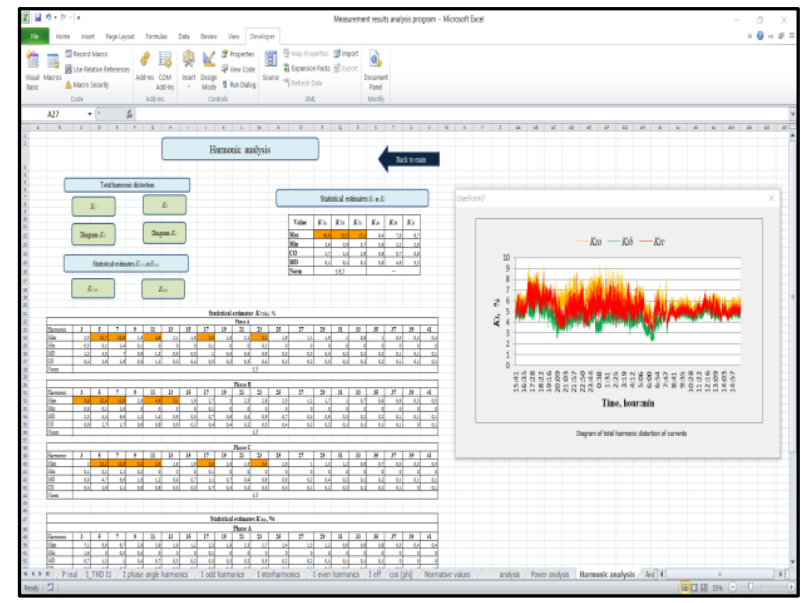

Fig. 4. Sheet 5 of the program for the analysis of measurement results.

Sheet 6 analyzes voltage and current interharmonics, for which the tables of statistical estimates are derived.

\section{Computer program for calculating the power of the active harmonic filter}

Based on the methodical approach presented above, the results of measurements of power quality indices and the "Steady-state allowable optimum operation mode" software package [13], we have developed the computer program for calculating the power of the active harmonic filter [14].

The computer program solves the optimization problem represented by the expressions (1) - (6). As the result of solving the optimization problem, the program determines the number of the filter installation node $\left(J_{A F}\right)$, the feeder transformer regulating tap voltage $\left(U_{K}\right)$, the active harmonic filter power $\left(S_{A F}\right)$, the minimum active power loss $\left(\Delta P_{\min }\right)$, and $\cos \varphi$. The flowchart of the program algorithm is shown in Fig. 5.

The flowchart uses the following notation: $Q_{A F}$ reactive power that should be generated by the active harmonic filter to compensate for reactive power of the load; $\Delta P_{1}$ - active power loss; $k$ - number of regulating taps of the feeder transformer; $\cos \varphi_{K}$ - load power factor, corresponding to $k$ - number of the regulating taps of the feeder transformer; $Q_{\min }, Q_{\max }$ - calculated reactive power corresponding to the range of change from the maximum to the minimum allowable value of $\cos \varphi_{K}$; $\Delta Q$ - increment of the reactive power of the active harmonic filter; $i$ - serial number of the network node from the array of candidate nodes for installation of the active harmonic filter; $I$ - total number of candidate nodes for installation of the filter.

The computer program is implemented in the MS Excel and Visual Basic programming environment for Windows applications. The program for calculating the power of the active harmonic filter consists of five sheets: Sheet 1: "Main"; Sheet 2: "Power of $Q_{A F}$ "; Sheet 3: "Power of harmonics $S_{n}$ ", Sheet 4: "Power of interharmonics $S_{m+1 / 2}$ ", Sheet 5: "Power of $S_{A F}$ ".

Fig. 6 shows the screenshot of the Sheet 1 of the program. In accordance with the commands of Sheet 1, we perform the input of the information for calculating the operation modes of the electrical network at the fundamental frequency, analysis of the input information, calculation of the electrical network mode to determine the losses of active power and the values of voltages at the nodes of the network.

The calculation is also performed for a comparative assessment of $\cos \varphi_{K}$ and $U_{K}$ values measured and calculated by means of the "Steady-state allowable optimum operation mode" software package at the node of the connection of the power supply system of the enterprise to the supply electrical network. Comparison of the calculated parameters with the results of measurements and their similar values attest to the correctness of the modeling of the electrical network of the enterprise.

The commands of Sheet 2 are used to calculate the value of load reactive power $\left(S_{Q}\right)$ that the active harmonic filter is to compensate for. In the process of calculating the losses of active power based on their minimum value, the program selects the node of the electrical network to install the active harmonic filter, if it is not designated.

Using the commands of Sheet 3 and Sheet 4 of the program we calculate the values of total apparent powers of harmonics $\left(S_{n}\right)$ and interharmonics $\left(S_{m+1 / 2}\right)$ that are to be eliminated by the active harmonic filter. To calculate the apparent power values, we make use of the results of measurements of the parameters of harmonic modes, interharmonics, and power quality indices at the node of the electrical network determined for the installation of the active harmonic filter. 


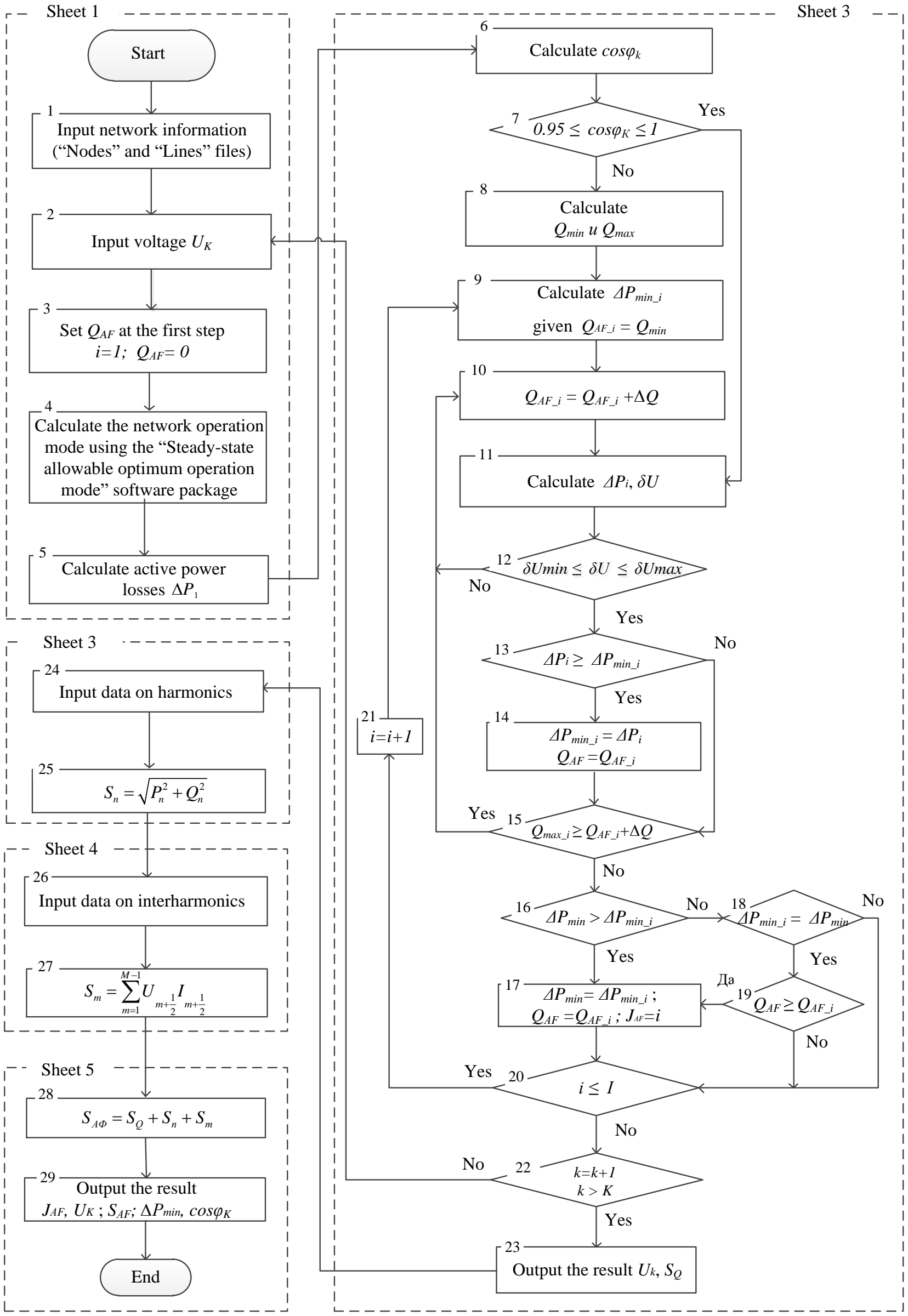

Fig. 5. Flowchart of the program algorithm. 


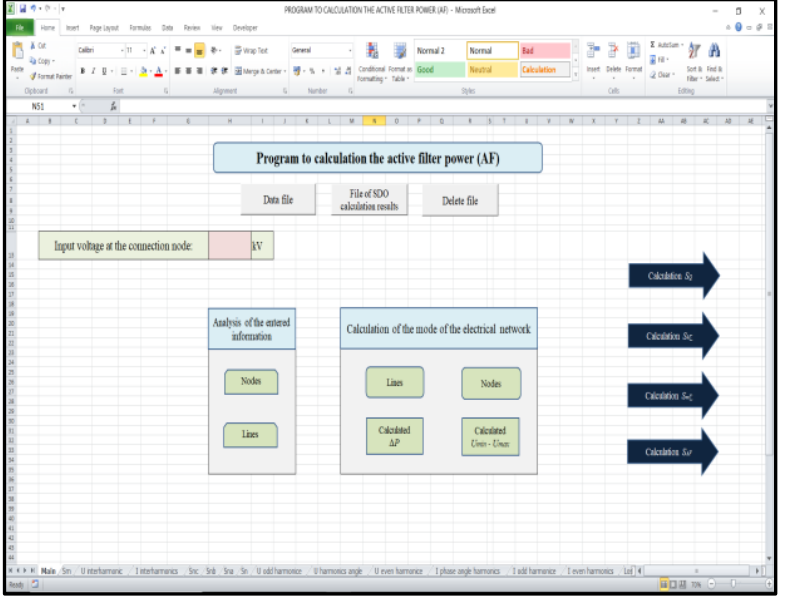

Fig. 6. Sheet 1 of the program for calculating the power of the active harmonic filter.

The commands of Sheet 5 of the program enable calculating the total apparent power of the active harmonic filter $S_{A F}$, designed to compensate for the reactive power of the load at the fundamental frequency and to eliminate harmonics and interharmonics. Sheet 5 of the program with the table of calculation results is shown in Fig. 7. The table shows the number of the network node to install the active harmonic filter, the value of the minimum active power losses, the load power factor, the reactive power of the active harmonic filter to compensate for reactive load power, the apparent power of harmonics, the apparent power of interharmonics, and the total apparent power of the active harmonic filter.

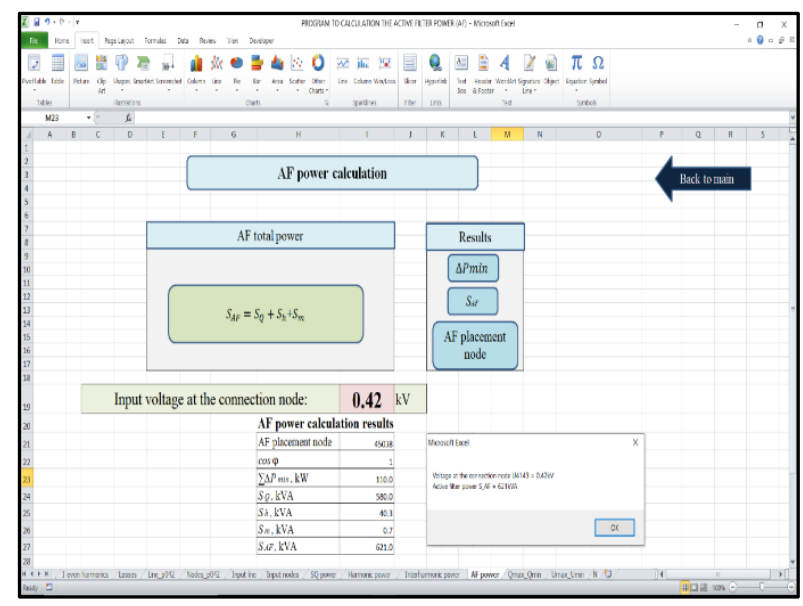

Fig. 7. Sheet 5 of the program for calculating the power of the active harmonic filter.

\section{Applying computer programs to determine the power of the active harmonic filter for the power supply system of the coal grading plant of the "Cua Ong-Vinacomin" company}

The measured information was processed and analyzed using the measurement analysis program. The results of processing and analysis are presented above in Tables 13. Table 1 shows the results of the analysis of $\delta U, K_{U}$, $\cos \varphi, K_{2 U}$ measured in three phases. Table 2 shows the results of the analysis of $K_{U(n)}, K_{I(n)}$ measured in three phases for eleven harmonics out of fifty at which the coefficients have the highest values. Table 3 shows the results of the analysis of $K_{U(m+1 / 2)}, I_{(m+1 / 2)}$ in three phases. According to the results of the analysis of power quality indices and operation mode parameters, it was decided to use the parallel active harmonic filter to solve the issues in the power supply system of the plant that were related to the power quality. With the help of the program for calculating the power of the active harmonic filter at the designated installation node 45038 , we have determined the regulating tap of the feeder transformer $-0.42 \mathrm{kV}$, the total apparent power of the active harmonic filter $-621 \mathrm{kVA}$, the load power factor at the node of the connection to the supply electrical network - 1 , and the minimum losses of active power in the electrical network of the coal grading plant $-110 \mathrm{~kW}$.

\section{Conclusion}

A methodical approach is presented for determining the power of the parallel active harmonic filter for power supply systems of industrial enterprises. Based on the methodical approach, computer programs have been developed to automate the process of calculating the power of the parallel active harmonic filter, which generates reactive power consumed by the load and eliminates harmonics and interharmonics of currents. Methodical approach and computer programs are used to determine the power of the active harmonic filter for the power supply system of the coal grading plant of the Vietnamese company "Cua Ong-Vinacomin".

The research was carried out under State Assignment Project (No. FWEU-2021-0001) of the Fundamental Research Program of Russian Federation 2021-2030.

\section{References}

1. GOST 32144-2013. Electrical energy. Electromagnetic compatibility of technical equipment. Power quality limits in public power supply systems, (2014).

2. National technical regulation on electrical engineering 2015/BCT - Part 1: Electricity network system (Vietnam, 2015).

3. L.I. Kovernikova, V.V. Sudnova, R.G. Shamonov et al. Power quality: current state, problems and proposals for solving them (Nauka, 2017).

4. D. Chapman, The Price of Poor Power Quality, Energosberezheniye, No. 1, (2004).

5. Zhezhelenko I.V., Sayenko Y.L., Electric losses, caused by high harmonics in electric power supply systems, Proceedings of X International School on 
Nonsinusoidal Currents and Compensation (ISNCC), Łagów, Poland, (2010).

6. R. Hartungi, L. Jiang, Investigation of power quality in health care facility, Proceedings of the International Conference on Renewable Energies and Power Quality, Granada, Spain, (2010).

7. G.M. Mustafa G.M., S.I. Gusev, Active filtercompensating devices of shunt and series type in electrical networks, Energetik, No. 8, (2019).

8. R.G. Shamonov, N.A. Alekseev, A.M. Matinyan, A.V. Antonov, Experience and prospects of using a high-voltage active filter of the MC series to improve the power quality, Elektroenergiya. Peredacha i raspredeleniye, No. 2 (65), (2021).

9. Gimeno-Sales F.J., Abellan Antonio, Achieving maximum efficiency in three-phase systems with a shunt active power compensator based on IEEE Std. 1459, IEEE Transactions on Industrial Electronics, Vol. 23, No. 2, (2008).

10. Wang Z., Wang Q., Yao W. and Liu J. A series active power filter adopting hybrid control approach, IEEE Transactions on Industrial Electronics, Vol. 16, No. 3, (2001).

11. Operating Manual Network Analyzer PQ-Box 150. Power-Quality Evaluation Software. URL: https://www.a-eberle.de/sites/default/files/media /BA_PQBox 150_EN_201803.pdf.

12. Certificate of state registration of the computer program No. 2021615347 "Program for analyzing the results of measurements of power quality indices and mode parameters". Date of registration in the Register of computer programs April 07, (2021).

13. Certificate of Conformity No. ROSS RU.SP20.H00008 "Software tool PS SDO-7 for analysis, optimization of steady-state modes and assessment of electricity losses in power plants" GOST R certification system Gosstandart of Russia.

14. Certificate of state registration of the computer program No. 2021613837 "Program for calculating the power of an active filter". Date of registration in the Register of computer programs March 16, (2021). 